\title{
A Computing Model of Artificial Intelligent Approaches to Mid-term Load Forecasting : a state-of-the-art- survey for the researcher
}

\author{
Pituk Bunnoon, Kusumal Chalermyanont, and Chusak Limsakul
}

\begin{abstract}
This article presents the review of the computing models applied for solving problems of midterm load forecasting. The load forecasting results can be used in electricity generation such as energy reservation and maintenance scheduling. Principle, strategy and results of short term, midterm, and long term load forecasting using statistic methods and artificial intelligence technology (AI) are summaried, Which, comparison between each method and the articles have difference feature input and strategy. The last, will get the idea or literature review conclusion to solve the problem of mid term load forecasting (MTLF).
\end{abstract}

Index Terms-Artificial Intelligent, Mid-term load, Forecasting, state-of-the-arts.

\section{INTRODUCTION}

The electricity is the necessity in the daily life and it is one of the main driving factors for country economic. In order to provide sufficient electricity and make the economic grown continuously, the load forecasting is required for the related electricity producers. Since, the construction of a power plant must take 5-10 years from planning, designing, environmental admitting to constructing step and there are few electric networks of Thailand and neighbor countries, the midterm load forecasting (MTLF) and the long term load forecasting (LTFL) are very important for building up the energy stability in Thailand [1,2].

Electricity load forecasting is not only significant for investment planning of three electricity authorities (Electricity Generating Authority of Thailand (EGAT), Metropolitan Electricity (MEA), and the Provincial Electricity Authority (PEA)) but also it is useful for estimating the financial statement of three electricity institutes. Figure1 shows the peak load profiles of the MEA and PEA classified by types of electricity consumers. The accuracy forecasted values make the proper investment for three electricity authorities. If the forecasted values are too

Manuscript received February 22, 2010.

P. Bunnoon is with Department of Electrical Engineering, Prince of Songkla University,Thailand (e-mail:add2002k@ hotmail.com) Ph.D. Prog.

$\mathrm{K}$. Chalermyanont is with Department of Electrical Engineering, Prince of Songkla University, Thailand (e-mail: kusumal.c@psu.ac.th).

C. Limsakul is with Department of Electrical Engineering, Prince of Songkla University, Thailand (e-mail: chusak.1@psu.ac.th). high, the exceeding investment is obtained and it will push this expenses to consumers. However, if the forecasted values are too low, the inadequate investment is occurred and it will cause electricity deficiency in the country.

The forecasted electricity demands are defined as two values: the peak value (Maximum load demand) and energy ${ }^{1}$ value (Electric energy demand).

The peak value is used in planning for new electricity plants while the energy value is used in planning for fuel providing. In the past, each electricity authority used the different methods to find these values.

Forecasting of the energy value

- The MEA and PEA uses the econometric model with Error Correction Model of Engle-Granger method or the econometric method with auto-Regressive Distributed Lag (ARDL) for monthly forecasting. The forecasting variables effected to electric load demands are electricity bill rate, GDP and temperature. Since, there are no monthly GDP data, they used the money quantity that circulate in people hand and the deposit reserve call of people in bank system instead adding with the specific losses in the distribution systems. The loss values in the distribution systems of the MEA and PEA are respectively $3.64 \%$ and $5.20 \%$ of the electricity demand taking from EGAT.

- Direct customers of EGAT use the direct inquires method from electricity consumers.

- The EGAT uses combining energy values from the MEA, the PEA and direct customers adding with the loss values in the generation system and transmission system to be the energy value of the system. The specific loss is about $5.10 \%$ of energy value of the system.

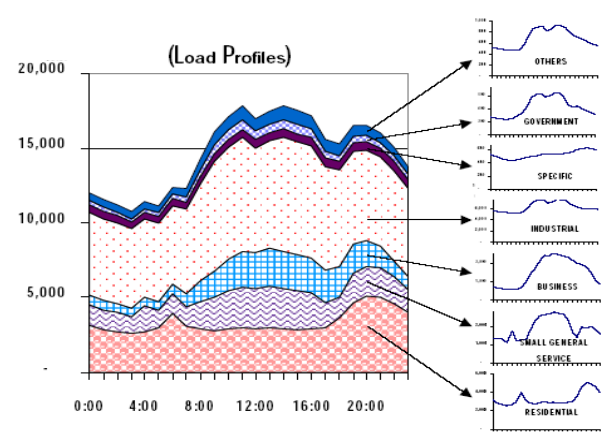

Figure.1: The use of Load Profile to find peak values of the MEA and the PEA[2].

Forecasting of the peak value 
- The MEA and PEA use the character of load profile of each customer to calculate energy value and adjust this value to be equal to the forecasted energy value of each type of customers. The over all peak value can be obtained by adding every load profiles of all customers as illustrated in Figure1.

- The direct customers of the EGAT use the principle of load profile by adjusting the load profile of each customer to be equal to the forecasted value and adding all load profiles to get the over all peak value.

- The EGAT adds all the load profile from The MEA and PEA as well as the direct customers to find the peak value. At the same time, this method can determine the peak value of the MEA, PEA and the direct customers in the same system (Coincident Peak) [2].

Beside the mentioned methods, the expert systems $[3,4,5,6,7]$ such as the artificial intelligent (AI) are frequently used for the system that required training and making decision based on the massive data. In the past, many artificial intelligence (AI) and Expert system (Es) methods such as artificial neural network (ANN), Fuzzy logic (Fs) and Genetic algorithm (GA) are proposed for the electricity load forecasting in short-term, mid-term and even long-term forecasting.

This paper presents a survey for a state of the art of load forecasting methods including input classifications, algorithm approaches and output determinations. The reviewed papers are covered in short-term, mid-term and long-term load forecasting. However, in last few sections, there will be emphasized on mid-term load forecasting. Load forecasting classification and paper reviewed are presented in section II. In section III, the mid-term load forecasting concepts are explained in details. The experimental and results analysis are described in section IV. Finally, the conclusion with some comparison are in section V.

\section{LOAD ForeCASTING ClASSIFICATION AND PAPER REVIEWED}

Load forecasting results have been used for operation planning of electric system as well as maintenance and fuel reserved planning [8].

The load forecasting can be classified into 3 different types according to the forecast period.

1. Short-term load forecasting,

2. Mid-term load forecasting,

3. Long-term load forecasting

In each load forecasting, period of time, forecasted values and aims of forecasting are noticeably different and they are comparably described in Table 1.

TABLE I. TYPES OF LOAD FORECASTING [8]

\begin{tabular}{llll}
\hline Forecast problem & Short term & Medium term & Long term \\
\hline Time horizon & $1 / 4-24 \mathrm{~h}$ & 1 day-few weeks & Few months-year \\
Forecasted value & Load curves & Load curves & Energy required \\
Accuracy & Exact load curves & Error $\ll$ Capacity & Exact energy \\
Time step & $1 / 4-1 \mathrm{~h}$ & $1 \mathrm{~h}$ & $1 \mathrm{~h}$ \\
Operation & Economic dispatch & Unit commitment & Reserve planning \\
Planning & Unit commitment & Reserve planning & Capacity expansion \\
\hline
\end{tabular}

Because of the difference of time period, forecasted values and aims of each load forecasting type, researchers in the past proposed many different algorithms and methods in order to obtain the precise load forecasting values. Next, relative papers and research topics of each load forecasting type are briefly concluded.

In 1987, [9] described about short-term load forecasting survey and comparing load forecasting in short-term,, midterm and long-term. In this paper, each research article has used differential techniques for determining the accurate output value. In [10-16], neural network for short-term load forecasting are used based on historical load and temperature input data. Moreover, some paper use additional input data from day types, humidity, wind speeds and seasons. This method is performed in compared with conventional method. Training network is achieved by supervise learning and back propagation algorithm. Another technique for short-term load forecasting is using fuzzy logic and neural network [17]. This paper presents that the neuro-fuzzy method that gives more accuracy results compared to one of the neural network method. In [18-19], types of input data using in fuzzy logic and neural network algorithms are historical load and weather. The case study is Electric company in China (Hang zhou Electric Power Company) In this paper, the principle of fuzzy rough sets is used to help neural network in forecasting. In [20], fuzzy logic with back propagation algorithm (BP) is used for short-term load forecasting in the uncertainty of the data input case. In this paper, the network composes of 51 inputs and 24 outputs and it is simulated by MATLAB . [21] presents short-term load forecasting by combining neural network and genetic algorithm with the case study in Taiwan while [22] presents the implementation of genetic algorithm method for fastening computation and increasing forecasting accuracy. The time period of this load forecast value is in 24 hours. In year 2001, [23] presents load forecasting model using the principle of wavelet decompositions to bring to more accuracy in electric load forecasting. In year 2006, [24] presents short-term load forecasting using fuzzy logic algorithm and input data of time and temperature. The input variable 'time' has been divided into eight triangular membership functions. The membership functions are Mid Night, Dawn, Morning, Fore Noon, After Noon, Evening, Dusk and Night. Another input variable 'temperature' has been divided into four triangle membership functions. They are Below Normal, Normal, Above Normal and High. The 'forecasted load' as output has been divided into eight triangular membership functions. They are Very Low, Low, Sub Normal, Moderate Normal, Normal, Above Normal, High and Very High. The case studies have been carried out for the Neyveli Thermal Power Station Unit-II (NTPS-II) in India. In 2004, [25] 


\section{ISSN: 1793-8236}

proposes a short term load forecasting using autoregressive integrated moving average (ARIMA) and artificial neural network (ANN) method based on non-linear load. It is concluded that using both methods can help each other in short-term load forecasting of the system. In 2007, [26] proposes a novel method approach to load forecasting using regressive model and artificial neural network (ANN model) with the case study carried out for Turkey. In this research, two methods are separately performed and compared. It shows that both methods give high accuracy results. In [2729], combination of artificial neural network (ANN), Genetic algorithm and Fuzzy logic (Fs) method are propsed for adjusting short-term load forecasting of electric system. Genetic algorithm is used for selecting better rules and back propagation algorithm is also for this network. The papers show that they give more accuracy results and faster processing than other forecasting methods. In 2005, [30] proposes short-term load forecasting for holiday by using fuzzy linear regression method. The proposed algorithm shows good accuracy and the average maximum percentage error of $3.57 \%$ in the load forecasting of the holidays. [31], in 2006, proposes a novel hybrid load forecasting algorithm, which combines the fuzzy linear regression method and the general exponential smoothing method with the analysis of temperature sensitivities. [32], in 2006, proposes the development of load forecasting which combines the fuzzy logic, neural network and chaos and another algorithm as shown in Figure 2. The proposed algorithm shows good accuracy or better than conventional method. [33] proposes an approach based on combined regression method and fuzzy inference system that developed for load forecasting. In addition, the fuzzy inference system makes a load correction inference from historical information and past forecast load errors from a multi linear regression model to infer a forecast load error. The effectiveness of the proposed approach to the short term load forecasting problem is demonstrated by practical data from the Taiwan Power Company. Paper [34] presents the development of a neuroexpert system for medium term load forecasting, back propagation algorithm is slightly modified and is used to train the artificial neural network. The proposed algorithm is tested on the practical $66 / 11 \mathrm{kV}$ primary distribution system of Mysore, Karnataka State, South India.

TABLE II. SUMMARY OF SHORT TERM LOAD FORECASTING CLASSIFIED BY ALGORITHM.

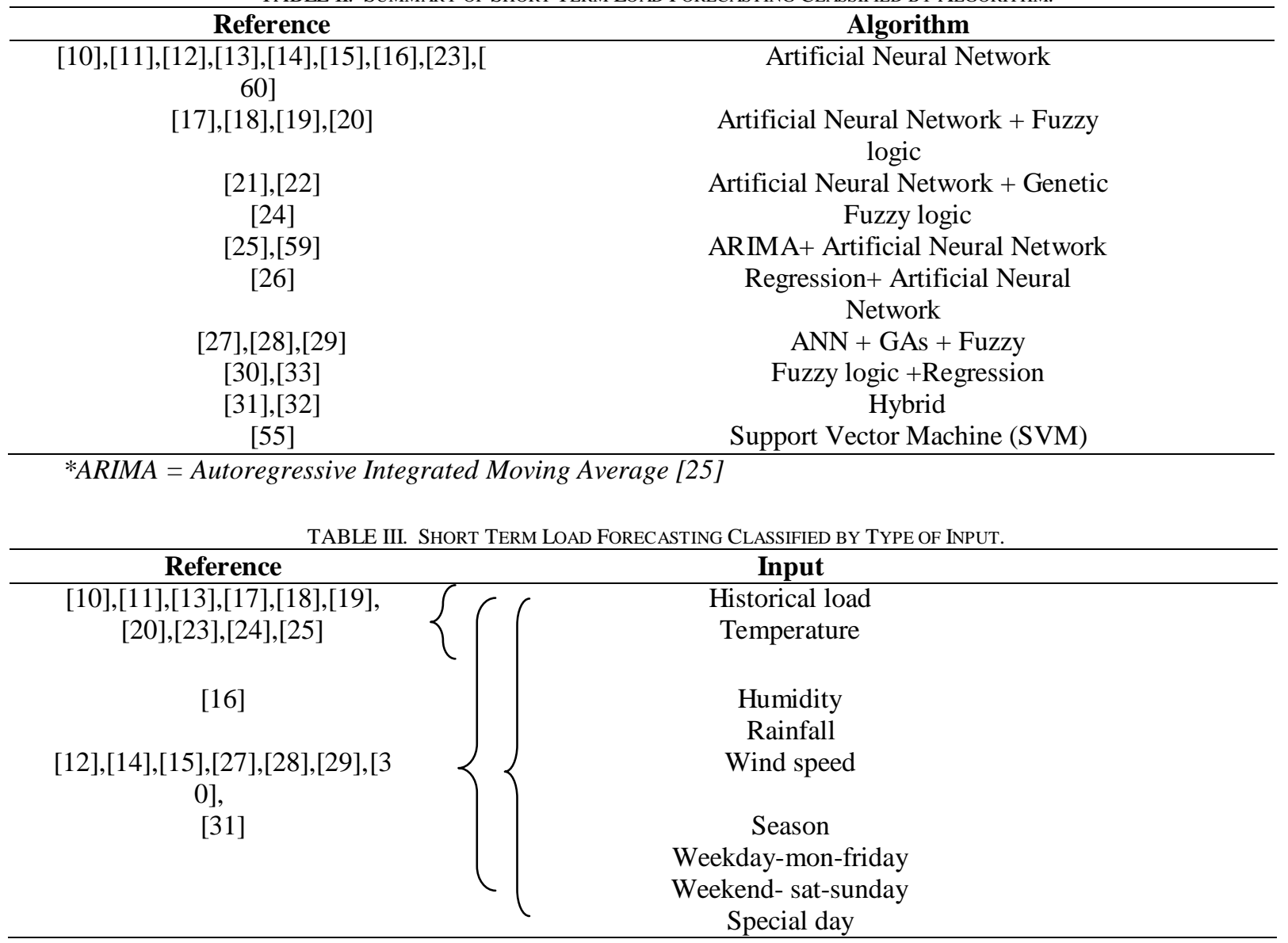

Form Table 2 ,it can see that the methods of load forecasting are mainly classified by algorithms. However, preceding article interests do not absolutely give the importance with algorithms. yet, they will also study in different kinds of the input variables in short term load forecasting such as temperature and load in the past etc. Other input variables of short term load forecasting are summarized in Table 3.
The pre-processing methods are used in some articles for selecting inputs before forecasting process. Many and different input data are chosen or grouped before the forecasting [17], [55], [42] The preprocessing methods are summarized in Table 4.

The previous summaries are the guidelines for midterm load forecasting that will be described in Section III. 
TABLE IV. SHORT TERM LOAD FORECASTING CLASSIFIED BY INPUT SELECTION METHODS

\begin{tabular}{cc}
\hline Reference & Pre-processing \\
\hline$[17],[55]$ & Self-Organizing map \\
{$[13]$} & Graphical modeling for selection input variable \\
{$[57]$} & Input dimension reduction \\
{$[42]$} & Fuzzy clustering \\
\hline
\end{tabular}

\section{The Mid-Term LOAd ForeCASTING CONCEPTS}

The articles of mid term load forecasting are summarized into 3 classification topics .

\section{A. Data Inputs}

\section{1) I nput classification}

Historical load inputs and meteorological data such as monthly maximum temperature, minimum temperature are used in [35-37] and [39, 40]. Economic variables are also included in [38]. The historical load data are collected from Electricity Generating Authority of Thailand (EGAT). Temperature data are provided from Meteorological Department and economic data are presented by the government of Thailand.

2) Input improvement

Articles [13, 17, and 42] proposed the input improvement or input selection for preprocessing of load forecasting. Many researchers used grouping data technique for decreasing complication of data and calculation time of the network. The preprocessing methods are for example SelfOrganizing Map and Data mining.

\section{B. Artificial Intelligence technology methods and other method for load forecasting}

1) Expert systems (Es)

Article [34], proposes neuro-expert for electric mid term load forecasting. The principle of Heuristic Rules is used for sorting out complex data can decreased the time and memory in training network.. Back propagation algorithm neural network is used for forecasting.

2) Artificial Neural Network (ANN)

[34-38] used Artificial Neural Network (ANN) approach to forecast electrical demand load, by using the data supporting from the government. The forecasting can be performed the results in yearly (to 15 years), weekly (to 3 years) and hourly (to 24 hours). ,Many groups of researchers used this forecasting approach for electricity mid term load forecasting. Also this method can be used for electricity peak load forecasting of distribution system [37]. By using the relationship of learning data in the past, present and the future of temperature, the network can forecast a daily peak load, total load of day and electricity monthly load consumption. However, in [38], historical load, economic and population variables are added for demand load forecasting by using neural network, time lagged feed forward network (TLFN).

3) Fuzzy logic $(F s)$

In [42], principle of fuzzy logic is integrated with another method, The fuzzy logic method is used to manage the datatolerance. In several articles used it for separating the input data [42]. Some articles use it in repeating process of forecasting in order to get the best answer. [17-19],[28-29]

\section{4) Genetic algorithms}

In [48-49], genetic algorithms are used for electric load demand forecasting. In [48], it is used together with neural network for load forecasting. This article is hourly load forecasting,. It is used as a base for monthly load forecasting, which duration time is 45 day and 49 day ahead by using historical data in 2005. In this method genetic algorithm is used to seek the initial weight of the neural network without random initial weight. It will give the network getting the results faster.

\section{5) Support Vector Machine (SVM)}

Support vector machine (SVM) is presented in [50] for electric load forecasting. This method used historical data in the past, present and the future in the weather and load, in 2001 to forecast load in 31 days ahead. This method is similar to neural network unless the support vector machine (SVM) can be used to separate input data before going to forecasting process.

\section{6) Statistics}

[51-53] proposes the methods of load forecasting by using the principle of mathematic or statistic such as Physical series algorithm [52] Autoregressive [51] and Nonlinear regression [53]. Statistics method is suitable for linear type of data. for example humidity, heat or temperature or meteorological parameter and historical monthly load [51].

\section{The adaptation of output forecasting}

In 1995, L.D.Voss, M.M.A.Salama, and J.Reeva [56] developed the forecasting technique to load forecasting by using neural network and output filter correction as shown in Figure 3 . In this article, MA Filtering is used to improve the output.

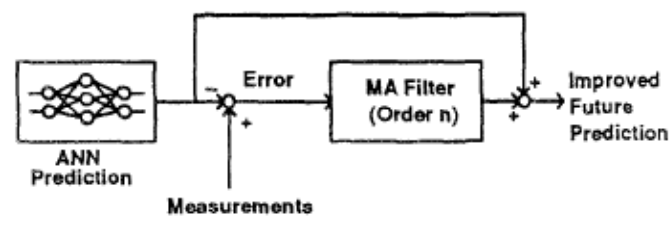

Figure.2: Load forecasting adaptation, MA Filtering [56]

\section{The Experimental Results And Analysis}

The articles mentioned previously can be comparably summarized in different points of view based on MAPE in Table 5-8. However, the comparison can not tell that which one of the method gives the best accuracy or which one is the best method. It is because each research technique is performed in different objectives and using different data. The forecasting will focus on the output error that can be determined as 


$$
M A P E=\frac{1}{N} \sum_{k=1}^{N}\left|\frac{X_{k}^{R}-X_{k}^{F}}{X_{k}^{R}}\right| .100
$$

Where

$$
\mathrm{N} \text { is number of test set }
$$

$$
\begin{aligned}
& X_{k}^{R} \text { is actual load of monthly load in k-th year } \\
& X_{k}^{F} \text { is parameter of forecasted in same year }
\end{aligned}
$$

\begin{tabular}{ccc} 
& & \\
\hline TABLE V. ACCURACY FOR EACH TYPE OF INPUT & Accuracy \\
\hline$[24]$ & Type of input & MAPE $<2 \%$ \\
{$[38]$} & Historical load, temperature & Can decrease \\
& Historical load, temperature, GDP, CPI, HIS, & error \\
& population & MAPE $<2 \%$ \\
& Historical load , temperature, humidity, wind speed, & \\
\end{tabular}

\begin{tabular}{cccc}
\hline$* G D P=$ Gross Domestic Product, CPI $=$ Current Price Index, HIS = Housing \\
\multicolumn{4}{c}{ TABLE VI. PrE-PROCESSING } \\
\hline Reference & Pre-processing & Algorithm & Accuracy \\
\hline$[41]$ & Knowledge based & ANN+ Knowledge & MAPE $2.29 \%$ \\
& & base & \\
{$[48]$} & Fuzzy clustering & Fuzzy+ANN & MAPE $1.568 \%$ \\
{$[55]$} & Self-Organizing map & SVM & MAPE (w) $1.65 \%(\mathrm{~s})$ \\
& & & $2.42 \%$
\end{tabular}

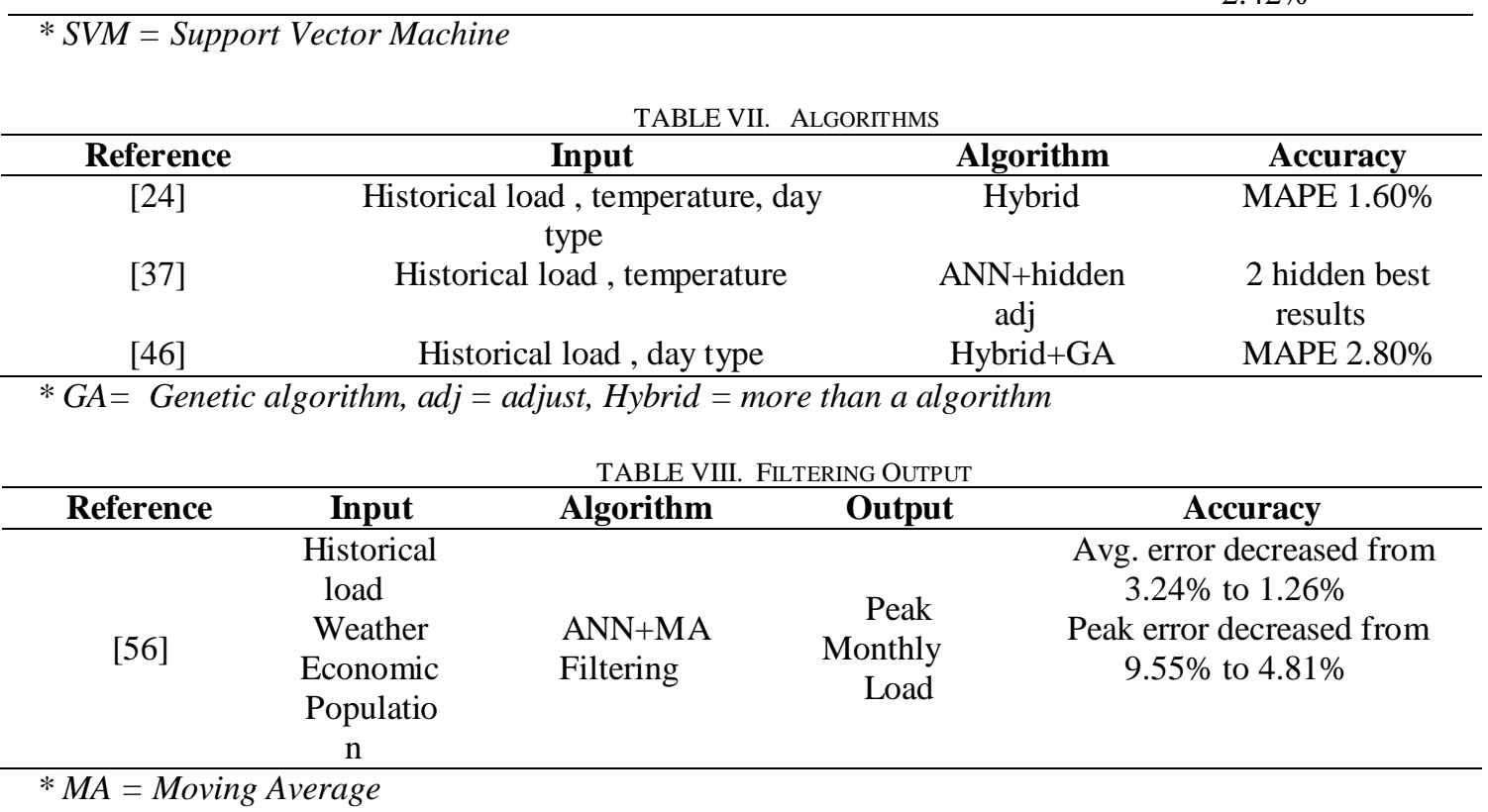

Table 5-8 show accuracy for each type of input, preprocessing, algorithm, and filtering output. The next, presents the article preceding of the forecasting from international research.

\section{CONCLUSION}

Mid term load forecasting (MTLF) becomes an essential tool for today power systems, mainly in those countries whose power systems operate in a deregulated environment. This kind of load forecast is useful for many applications such as maintenance scheduling and development of cost efficient fuel purchasing strategies. In the past load forecasting are performed using the principle of artificial intelligence technology. Each method uses difference input and gives difference accuracy depending on the complexity of input.

The artificial intelligence technology is used as a decision part in mid term load forecasting. It has ability to work with non-linear data. Moreover, it can be effectively performed in complicate forecasting model for continuous data or signal. This technology will help the conventional method (statistical) in the complexity problems based on the value of the variable between the variable input and nonlinear correlation by training of data, learning process.

\section{ACKNOWLEDGE}

This work was granted (funded) by Office of the Higher Education Commission. Pituk Bunnoon was supported by CHE510382 Ph.D. Scholarship. I would like to thank the Thai meteorological department, Ministry of commerce Thailand, the office of the nation economic and social development board, and Organization of electricity generating authority of Thailand (EGAT) for data information and thank assistance Professor Dr. Kusumal Chalermyanont and associate Professor Dr.Chusak Limsakul 
advisors for clarifying several points in my research.

\section{REFERENCE}

[1] Energy to Energy Policy and Planning Office, Ministry of Energy, Royal Thai Government, Thailand.

[2] Energy to Energy Policy and Planning Office, "Load forecasting reports 2547," Ministry of Energy, Royal Thai Government, Thailand.

[3] Peter Vas., "Artificial-Intelligence-Based Electrical Machines and Drives," Oxford science publication, 1999.

[4] Lefteri H.Tsoukalas, Robert E.Uhrig, "Fuzzy and Neural Approaches in Engineering," A wiley-Interscience publication, JohnWilley\&Sons INC, 1997.

[5] Peter M.ills, Albert Y.Zomaya, and Moses O.Tade, "Neuro-Adaptive Process Control A Practical Approach,” John Willey\&Sons Ltd, 1996.

[6] R.A. Aliev, R.R.Aliev, "Soft Computing and its Application," World scientific publication, 2001

[7] Stamatios V.Kartalopoulos.Understanding, "Neural Network and Fuzzy logic Basic Concepts and Applications," AT\&T, 1996.

[8] K. Metaxiotis, A. Kagiannas, D. Askounis, J. Psarras, "Artificial intelligence in short term electric load forecasting :a state-of-the-art survey for the research," Energy Conversion and Management 44, 2003, pp.1525-1534.

[9] Georgr gross,franscisco D.galiana, "Short-term load forecasting," Preceeding of the IEEE, vol.75,no.12, December 1987, pp.1558-1573.

[10] Tomonoobu senjyu, hitoshi takara,katsumi ueezato,toshihisa funabashi, "One-hour ahead load forecasting using neural network," IEEE Trans. power syst., vol.17, no.1, February 2002, pp.113-118.

[11] Zhiyong Wang,Yijia Cao, "Mutual Information and Non-fixed ANNs for Daily Peak load forecasting," Power Systems Conference and Exposition, IEEE PES ,2006, pp:1523 - 1527.

[12] wenjin Dai, ping Wang, "Application of pattern recognition and artificial neural network to load forecasting in electric power system," Third international conference on natural computation, ICNC 2007.

[13] Hiroyuki mori,eitaro kurata, "Graphical Modeling for Selecting Input Variables of Short-term Load Forecasting," Power Tech, 2007 IEEE Lausanne, July 2007, pp:1084 - 1089.

[14] G.A.Adepoju, S.O.A.ogaunjuyigbe, and K.O.Alawode, "Application of neural network to load forecasting in nigerian electrical power system," The pacific journal of science and technology, vol.8, no.1, May 2007, pp.68-72.

[15] Mohsen Hayati, Yazdan Shirvany, "Artificial neural network approach for short term load forecasting for Illam region," International journal of electrical, computer, and system engineering, vol.1, no. 2, pp.121-125.

[16] H.A.Salama, A.F.AbdElGawad, H.M.Mahmond, E.A.Mohamed, S.M.Saker, "Short term load forecasting investigations of eqyptian electrical network using ANNs," Universities Power Engineering Conference, 2007, pp:550 - 555.

[17] Bhavesh kumar chauha, amit sharma, and m.hanmandlu, "Neurofuzzy approach based short term electric load forecasting," 2005 IEEE/PES Transmission and Distribution Conference \& Exhibition, Asia and Pacific Dalian, Chaina.

[18] Z.Y.Wang, C.X.Guo, Y.J.Cao, "A New method for short term load forecasting integrating fuzzy tough sets with artificial neural network," Power Engineering Conference, IPEC 2005, pp:1 - 173.

[19] Cuiru wang, Zhikun cui , Qi chen, "Short term load forecasting based on fuzzy neural network," Intelligent Information Technology Application, Workshop on 2-3 Dec. 2007, pp:335 - 338.

[20] Hari seetha and R.saravanan, "Short term electric load prediction using fuzzy BP," Journal of computing and information technologyCIT 15, 2007, pp:267-283.

[21] Chih-hsien kung, michael J.Devaney, chung-ming huang, chih-ming kung. An adaptive power system load forecasting scheme using a genetic algorithm embedded neural network. IEEE Intrumentation and Measurement technology conference St.Paul,Minnesota, USA, May 18-20.

[22] L.L.Lai,H.Subasinghe, N.Rajkumar, E.Vaseekar, B.J.Gwyn, V.K.Sood, "Object oriented genetic algorithm based artificial neural network for load forecasting," Springer-verlag berlin ,1999, pp:462469.

[23] Zhao-yang dong, Bai-ling zhang, Qian huang. A daptive neural network short term load forecasting with wavelet decompositions. IEEE Porto power tech conference, 10-13 september,porto,Portugal.
[24] S.Chenthur Pandian, K.Durais wamy, C.Christer Asir Rajan, N.Kanagaraj, "Fuzzy approach for short term load forcasting," Electric Power Systems Research 76, 2006, pp:541-548.

[25] Jian-Chang Lu, Dong-xiao niu, zheng-yuan jia, "A study of short term load forecasting based on arima-ann," Machine Learning and Cybernetics, vol.5, 2004, pp:3183 - 3187.

[26] Ummuhan basaran filik, Mehmet kurban, "A new approach for the short term load forecasting with autore gressive and artificial neural network models," International journal of computational intelligence research, vol.3, no.1, pp:66-71.

[27] P.K.Dash, S.Mishra, S.Dash, A.C.Liew, "Genetic optimization of a self organizing fuzzy-neural network for load forecasting," IEEE 2000.

[28] Gwo-ching liao, Ta-peng tsao, "Integrated genetic algorithm/Tabu search and neural fuzzy networks for short-term load forecasting," Power Engineering Society General Meeting, 2004, pp:1082 - 1087

[29] Gwo-ching liao, Ta-peng tsao, "Novel GA-Based Approach and Neural fuzzy networks application in short-term load forecasting," Power Engineering Society General Meeting, 2004, pp:589-594

[30] Kyung-bin song, young-sik baek, dug hun hong and gilsoo jang, "Short-term load forecasting for the holidays using fuzzy linear regression method" IEEE Trans. power syst., vol.20, no.1, 2005, pp:96-101

[31] Kyung-bin song, seong-kwan ha, jung-wook park, dong-jin kweon, kyu-ho kim, "Hybrid load forecasting method with analysis of temperature sensitivities," IEEE Trans. power syst., vol.21, no.2, 2006, pp:869-876.

[32] Gwo-ching liao, Ta-peng tsao, "Application of a fuzzy neural network combined with a chaos genetic algorithm and simulated annealing to short term load forecasting," Evolutionary Computation, vol.10, issue 3, 2006, pp:330 - 340 .

[33] R.H.Liang and C.-C.Cheng, "Combined regression-fuzzy approach for short-term load forecasiting," Generation, Transmission and Distribution, vol.147, issue 4, 2000, pp:261 - 266.

[34] Adiga S. Chandrashekara, T. Ananthapadmanabha, A. D. Kulkarni. A neuro-expert system for planning and load forecasting of distribution systems. International Journal of Electrical Power \& Energy Systems, vol.2, issue 5, 1999, pp:309-314.

[35] J.V.Ringwood, D.Bofelli and F.T.Murray, "Forecasting electricity demand on short, medium and long time scales using neural networks," Journal of Intelligent and Robotic System 31, 2001, pp:129-147.

[36] Ioan Borlea, Adrian Buts, "Some aspects concerning mid term monthly load forecasting Using ANN," EUROCON , 22-24 Nov.2005.

[37] T.Yalcinoz, U.Eminoglu, "Short term and medium term power distribution load forecasting by neural networks," Energy Conversion and Management 46, 2005; pp:1393-1405.

[38] Danilo Bassi, Oscar Olivares, "Medium term electric load forecasting using TLFN neural networks," IJCCC, vol.I, no.2, 2006, pp:23-32.

[39] M.Ghiassi, David K.Zimbra, H.Saidane, "Medium term system load forecasting with a dynamic artificial neural network model," Electrical Power system Research 76, 2006, pp:302-316

[40] M.C.Falvo, R.Lamedica, "Meteorological parameters influence for medium term load forecasting," 2006 , pp:1296 - 1301.

[41] M.C.Falvo, R.Lamedica, "A Knowledge based system for medium term load forecasting," IEEE, 2006, pp:1291 - 1295.

[42] Lu Yue, Yao Zhang, Huifan Xie, Qing Zhong, "The fuzzy logic clustering neural network approach for middle and long term load forecasting, Grey Systems and Intelligent Services(GSIS 2007), 2007, pp:963 - 967.

[43] Nima Amjady, Farshid Keynia, "Mid-term load forecasting of power system by a new prediction method," Energy conversion and management (science direct),2008, pp.1-10.

[44] E.Gonzalez-Romera,M.A.Jaramillo-Moran,and D.CarmonaFernandez, "Monthly electric energy demand forecasting with neural networks and fourier series," Energy conversion and management (science direct),2008, pp:1-8.

[45] R.E.Abdel-Aal, "Univariate modeling and forecasting of monthly energy demand time series using abductive and neural networks," Computer \& Industrial Engineering 54, 2008, pp:903-917.

[46] Ronaldo R.B. de Aquino, Aida A.Ferrira, Manoel A.Carvalho Jr, "Development of a hybrid intelligent system for electrical load forecasting," IBERAMIA-SBIA, 2006, pp:228-237.

[47] Ronaldo R.B. de Aquino, Aida A.Ferrira, Manoel A.Carvalho Jr., "Combining Artificial neural networks and heuristic rules in a hybrid intelligent load forecast system," ICANN, 2006, pp:757-766. 
[48] Ronaldo R.B.de Aquino, Otomi Nobrega Neto, Milde M.S.Lira,Aida A.Ferreira,Manoel A, "Development of an artificial neural network by genetic algorithm to mid term load forecasting," Neural Networks 2007(IJCNN 2007). International Joint Conference, 2007, pp:1726 1731.

[49] Ronaldo R.B.de Aquino, Otomi Nobrega Neto, Milde M.S.Lira, Aida A.Ferreira, Manoel A., "Using Genetic Algorithm to Develop a Neural network based load forecasting," ICANN, 2007, pp:738-747.

[50] M Gavrilas, I Ciutea and C Tanasa, "Load Forecasting Using support vector machines," IEE 2001,18-21 June 2001, pp:1-7.

[51] S.Mirasgedis, Y.Sarafidis, E.Georgopoulou, D.P. Lalas, "Models for mid-term electricity demand forecasting incorporating weather influences," energy 31,2006, pp:208-227.

[52] Xie Da, Yu Jiangyan, Yu Jilai, "The physical series algorithm of midlong term load forecasting," Electric power research .53, 2000, pp:3137.

[53] Alexander Bruhns, Gilles Deurveilher, Jean-sebastien roy, "A nonlinear regression model for mid-term load forecasting and improvements in seasonality," 15th PSCC Liege, August 2005.

[54] G.J.Tsekouras, P.B.Kotoulas, C.D.Tsirekis, E.N.Dialynas, N.D.Hatziargyriou, "A pattern recognition methodology for evaluation of load profiles and typical days of large electricity customers," Electric power systems research78, pp:1494-1510.

[55] Shu fan, Chengxiong Mao, Luonan Chen, "Electricity Peak Load Forecasting with Self-Organizing Map and Support Vector Regression," Transactions on Electrical and Electronic Engineering, 2006, pp:330-336.

[56] L.D. Voss , M.M.A. Salama, J.Reeve, "A Practical Approach to Electric Load Forecasting Using Artificial Neural Networks with Corrective Filtering," IEEE, 1995.

[57] Xu Tao, He Renmu, Wang Peng and Xu Dobgjie, "Input dimension reduction for load forecasting based on support vector machines," IEEE International Conference on Electric Utility Deregulation ,April 2004, Hong Kong.

[58] A.A. El Desovley and M.M. ElKateb, "Hybrid adaptive techniques for electric load forecasting using ANN and ARIMA," IEE proceeding, vol.147, no.4, July 2000.

[59] Pituk Bunnoon, Kusumal Chalermyanont, and Chusak Limsakul, "Mid Term Load Forecasting of the Country Using Statistical Methodology: Case study in Thailand," 2009 International Conference on Signal Processing System, pp.924-928, May 2009.

[60] Pituk Bunnoon, Kusumal Chalermyanont, and Chusak Limsakul, "Peak load demand forecasting using 2-level discrete wavelet decomposition and neural network algorithm," Proceeding of SPIE, second international conference on digital image processing, Feb.26$27,2010$.

Pituk Bunnoon received the B.S. degree from King Mongkut's Institute of techhnology Ladkrabang, Thailand, in 1998, and the M.S. degree in electrical engineering from Prince of Songkla University, Thailand, in 2004 His research interest is application of artificial intelligence to power system planning and operation.

Kusumal Chalermyanont received the B.S. degree from Prince of Songkla University, Thailand, in 1993, the M.S. degree in electrical engineering from University of Colorado at Boulder in 1999, and the Ph.D. in electrical electrical engineering from University of Colorado at Boulder in 2003. Her research interest are power electronics, magnetic designs for power electronics, renewable energy system/management.

Chusak Limsakul received the B.S. degree from King Mongkut's Institute of technology Ladkrabang, Thailand, in 1978, and the D.E.A. degree from INSAT France, in 1982, and Docteur Ingenieur form INSAT France, in 1985. His research interest are digital signal processing, sensors and instrumentations, and automation. 